\title{
Magnetic shepherding of nanocatalysts through hierarchically- assembled Fe-filled CNTs hybrids
}

Michele Melchionna, ${ }^{a}$ Alessandro Beltram, ${ }^{a}$ Antoine Stopin, ${ }^{b}$ Tiziano Montini, ${ }^{\mathrm{a}, \mathrm{c}}$ Rhys W. Lodge, ${ }^{\mathrm{d}}$ Andrei N. Khlobystov, ${ }^{d}$ Davide Bonifazi, ${ }^{* b}$ Maurizio Prato ${ }^{*}, \mathrm{e}, \mathrm{f}$ and Paolo Fornasiero ${ }^{*}, \mathrm{c}$

[a] Dr. M. Melchionna, Dr A. Beltram, Dr. T. Montini, Prof. Dr. P. Fornasiero, Prof. Dr. M. Prato

Department of Chemical and Pharmaceutical Sciences, University of Trieste and INSTM Trieste Research Unit, Via L. Giorgieri 1, 34127 Trieste, Italy. Emails: prato@units.it, pfornasiero@units.it

[b] Dr. A. Stopin, Prof. Dr. D. Bonifazi

School of Chemistry, Cardiff University, Park Place, Main Building, CF10 3AT, Cardiff, United Kingdom. Email: bonifazid@cardiff.ac.uk

[c] Prof. Dr. P. Fornasiero, Dr T. Montini

ICCOM-CNR URT, University of Trieste, Via L. Giorgieri 1, 34127 Trieste, Italy

[d] R.W. Lodge, Prof. Dr. A.N. Khlobystov, School of Chemistry, University of Nottingham, University Park, Nottingham NG7 2RD, United Kingdom.

[e] Prof. Dr. M. Prato

Carbon Nanobiotechnology Laboratory, CIC biomaGUNE, Paseo de Miramón 182, 20009 Donostia-San

Sebastian (Spain).

[f] Prof Dr. M. Prato

Ikerbasque, Basque Foundation for Science, 48013 Bilbao (Spain).

Abstract: Mechanically robust, chemically stable and electronically active carbon nanotubes (CNTs) are widely used as supports in catalysis. Synergistic effects between CNT and the active phase critically depend on the homogeneity of the carbon/inorganic interface, whose assembly is difficult to achieve without admixtures of free-standing inorganic matrix. Here we show that Fe-filled CNTs, employed as nanocatalyst supports, allow a facile preparation of highly pure and uniform CNT/nanocatalyst materials, by taking advantage of magnetic separation from poorly-defined components (e.g. aggregates of inorganic nanocatalysts). The higher homogeneity translates into higher catalytic activity in two industrially important processes: the photocatalytic hydrogen production and the water-gas shift reaction, WGSR (increase of $\sim 48 \%$ activity for the former and up to $\sim 45 \%$ for the latter as compared to catalysts isolated by standard filtration). In addition, the magnetic Fe core in the nanotubes enables effective separation and re-use of the nanocatalyst without loss of activity. This study demonstrates significant potential of magnetic CNTs as next generation of sustainable catalyst supports that can improve production of hydrogen and reduce the use of precious metals. 
Keywords: carbon nanotubes $\bullet$ hierarchical nanostructures $\bullet$ hydrogen evolution $\bullet$ water-gas shift $\bullet \mathrm{Pd}$ nanoparticles $\bullet$ magnetic nanomaterials

\section{Introduction}

The introduction of a precise hierarchical order in the assembly of nanostructured materials is nowadays considered as the latest frontier in heterogeneous catalysis. Energy-related applications have particularly profited from the use of this concept [1, 2]. The structural interplay of hierarchically organized phases is often the key factor for the achievement of notable boosts of catalytic performance in several chemical transformations [3]. In many cases, such interplay crucially depends on the interface, in particular a tight contact between the different components, which secures an efficient electronic communication and also underpins an enhanced robustness of the catalytically active sites [3]. The assembly of metal/metal oxide core-shell systems, where metal nanoparticles are enveloped within a layer of metal oxide phase, is an excellent demonstration of this important concept, showing improved activity and stability, arising from the enhanced contact between the two components [4].

Carbon nanotubes represent a very useful scaffold in modern nanostructured catalysts [5], having been combined with a range of inorganic counterparts, resulting in hierarchical nanohybrids with intriguing catalytic features [6]. For example, in photocatalytic processes the presence of CNTs in tight contact with the photoactive phase retard the recombination of the photogenerated holes and electrons, thus generating longer lived charge separated states, thanks to the electron scavenging ability of the graphitic scaffold [7]. On the other hand, in the WGSR, CNTs may help the electronic suppression of the deactivation pathway, with the catalytic performances depending on an optimum coverage of the carbon component with the active metal/metal oxide phase [8]. Many other examples are available where the beneficial role of CNTs is proven, and the critical importance of a close electronic crosstalk with the metal phase substantiated [9]. Despite the considerable advances in the preparation of CNT nanohybrids, a central and recurrent drawback lies in the generally poor control over the synthetic route to secure effective formation of a homogeneous nanohybrid. In fact, synthesized CNTs-inorganic hybrids generally present considerable amounts of free-standing inorganic phase that [10], possessing lower activity, diminishes the catalytic efficiency of the hybrid materials. An attempt to reduce the admixtures of metal phase by simply decreasing the metal loading is not viable, as the coverage of the CNTs and the isolated aggregates formation proceed in parallel, and a decrease of the metal loading also leads to 
partly bare CNTs with resulting lowering of catalytic activity $[8,11]$. The development of a general and efficient strategy to maximize the interfacing between the CNTs and the inorganic active components while reducing the total amount of the metal phase would guarantee a step forward for the definitive establishment of CNT-based hybrid nanocatalysts. We conjectured that the shepherding of the more active CNT/metal nanostructures, liberated from post-synthetic CNT-free aggregates, could be the winning strategy, maximizing the catalytic potential by full exploitation of the CNT-inorganic phase interaction.

For their part, magnetic nanocatalysts have become an appealing alternative to standard catalysts, as they bring the great advantage of avoiding cost- and time-consuming protocols for filtration, separation and isolation from by-products [12-14] and an extra asset in the ease of the post-catalytic recovery step [15]. Some of us have previously reported the synthesis and characterization of multi-walled CNTs (MWCNTs) filled with a magnetic Fe phase (Fe@CNTs) [16] for use in cancer treatment and cell shepherding $[17,18]$ as well as in electrocatalytic $\mathrm{O}_{2}$ reduction and $\mathrm{H}_{2}$ evolution [19]. Building on these concepts, here we describe the hierarchical assembly of Fe@CNTs/Pd@MO ${ }_{2}$ nanohybrid systems, integrating magnetic Fe@CNT with an inorganic matrix made of Pd nanoparticles embedded within $\mathrm{TiO}_{2}$ or $\mathrm{CeO}_{2}$ nanocrystals. The magnetic Fe@CNTs core can harness a general and simple sieving process to obtain the most active CNT-based catalyst package as demonstrated by the measuring of the catalytic performances in two important energy-related processes: $\mathrm{H}_{2}$ photocatalytic evolution and water-gas shift reaction (WGSR). Moreover, extension of the application of these nanohybrids to other chemical transformations may imply a direct involvement of the endohedral Fe phase, which can facilitate charge transfer pathways $[20,21]$. As a further asset of these new CNT-based hybrids, the recyclability of the catalyst can rely on the same simple principle of magnetic separation. We predict that the generality of our finding could spark new research on the use of a next generation of magnetic CNTs for heterogeneous catalytic applications.

\section{Experimental}

\subsection{Synthesis of Fe@CNTs}

The preparation of Fe@CNTs followed the procedure previously developed [19]. In detail: in a $180 \mathrm{~cm}$ long quartz tube of a furnace setup, a $10 \mathrm{~cm}$ long quartz substrate was introduced in the temperature region around $900{ }^{\circ} \mathrm{C}$ and a constant flow of $0.2 \mathrm{~m}^{3} \mathrm{~h}^{-1}$ of argon was used to purge the system of remaining air. Under this flux, ferrocene ( $5 \mathrm{~g}$ ) was introduced in a boat and placed in a region close to 
$550{ }^{\circ} \mathrm{C}$. Toluene vapour was then flown into the quartz tube of the furnace by the argon purge of $0.2 \mathrm{~m}^{3}$ $\mathrm{h}^{-1}$ for 15 minutes. The toluene flow is then turned off and the quartz substrate is moved to a cool zone of the $180 \mathrm{~cm}$ long quartz tube to allow the sample to cool down to room temperature under an argon flux of $0.2 \mathrm{~m}^{3} \cdot \mathrm{h}^{-1}$. The black material is then washed with ethanol to remove any residual ferrocene to obtain pristine Fe@CNTs (about $2.3 \mathrm{~g}$ ). The material was then subjected to $\mathrm{HCl}$ washing in order to remove any external Fe or Fe oxide. As produced Fe@MWCNTs $(250 \mathrm{mg}$ ) was introduced into a $1 \mathrm{~L}$ round-bottomed flask with methanol $(375 \mathrm{~mL})$. The reacting mixture was sonicated for 10 min and an aqueous $\mathrm{HCl}$ solution $(4 \mathrm{M}, 125 \mathrm{~mL}$ ) was added and the mixture was sonicated for 10 min and stirred for $24 \mathrm{~h}$ at room temperature. Water $(375 \mathrm{~mL})$ was added and the mixture filtered through a $0.45 \mu \mathrm{m}$ hydrophobic polytetrafluoroethylene filter. The precipitate was redispersed by 10 min of sonication in $100 \mathrm{~mL}$ of methanol, filtered through the same filter and washed with water (100 mL). This procedure was repeated twice. The precipitate obtained was then washed with methanol (few $\mathrm{mL}$ ). A black powder (about $220 \mathrm{mg}$ ) was finally recovered.

\subsection{Functionalization of Fe@CNTs with benzoic acid (f-Fe@CNTs)}

Fe@CNTs (100 mg) were placed in a $500 \mathrm{~mL}$ round bottomed flask and N-methyl-2-pyrrolidone (NMP, $67 \mathrm{~mL}$ ) was added. The solution was sonicated for $20 \mathrm{~min}$ and p-amino-benzoic acid (675 $\mathrm{mg}$ ) was introduced, the solution was sonicated for another $10 \mathrm{~min}$ and diisopropylethylamine (DIPEA, $40 \mu \mathrm{L}$ ) was added. After $10 \mathrm{~min}$ of further sonication, isoamyl nitrite $(1.5 \mathrm{~mL})$ was added slowly and the reaction mixture was stirred at $90{ }^{\circ} \mathrm{C}$ for $20 \mathrm{~h}$. The dispersion was cooled to room temperature and was filtered through $0.45 \mu \mathrm{m}$ hydrophobic polytetrafluoroethylene filters. The precipitate was redispersed by $10 \mathrm{~min}$ of sonication in $100 \mathrm{~mL}$ of ethyl acetate and filtered through the same filter. This step was reproduced two additional times and the final precipitate was dispersed in $100 \mathrm{~mL}$ methanol by 10 min sonication, water $(200 \mathrm{~mL})$ was added and the suspension was filtered through the same filter. Two additional washings with water $(50 \mathrm{~mL})$ were also realized. The precipitate was finally washed with few $\mathrm{mL}$ of methanol to facilitate drying. About 85 mg of black powder (f-Fe@CNTs) were thus recovered.

\subsection{Synthesis of Fe@CNTs/Pd@TiO 2-filt and Fe@CNTs/Pd@TiO 2-magn}

The precursor Pd-MUA (MUA = mercapto-undecanoic acid) was prepared according to a previous procedure [22]. The $f$-Fe@CNTs were dispersed in absolute ethanol (EtOH mL/f-Fe@CNTs mg ratio: 2.5) by sonication for 30 minutes meanwhile a Pd-MUA THF solution (containing $1.5 \mathrm{mg}$ of Pd) was slowly added to a THF solution of $\mathrm{Ti}(\mathrm{OBu})_{4}$ (containing nominal $88.5 \mathrm{mg}$ of $\mathrm{TiO}_{2}$ respectively). Then the ${\mathrm{Pd} @ \mathrm{TiO}_{2}}_{2}$ 
precursor solution was slowly added under sonication to the $f$-Fe@CNTs dispersion and the mixture was further sonicated for 30 minutes. Finally a $10 \%$ solution of $\mathrm{H}_{2} \mathrm{O}$ in $\mathrm{EtOH}\left(\mathrm{Ti}(\mathrm{OBu})_{4} / \mathrm{H}_{2} \mathrm{O}\right.$ molar ratio: 1/120) was dropped and the mixture sonicated for 30 minutes. The materials were collected by either magnetic separation (Fe@CNTs/PdTiO ${ }_{2}$-magn) or by filtration (Fe@CNTs/PdTiO 2 -filt). For the former, the dispersion was left in a vial close to a NdFeB magnet (purchased by www.supermagnete.it) for 3 hours, the liquid phase was then discarded. This operation was repeated twice redispersing the material in fresh $\mathrm{EtOH}$. The solid was then recovered and left to dry. For the filtration method, the dispersion was filtered on a $0.45 \mu \mathrm{m}$ polytetrafluoroethylene Millipore membrane, washed with ethanol and dried overnight at $80{ }^{\circ} \mathrm{C}$. The solids were thermally treated under a flow of $\mathrm{H}_{2}(10 \%)$ in Argon (total flow 220 $\mathrm{mL} \mathrm{min}^{-1}$ ) for 5 hours at $450{ }^{\circ} \mathrm{C}$.

\subsection{Synthesis of Fe@CNTs/Pd@CeO 2 -filt and Fe@CNTs/Pd@CeO - -magn}

The synthesis was similar to the previous one used for $\mathrm{TiO}_{2}$, with the exception that the Ce precursor was $\mathrm{Ce}(\mathrm{ODe})_{4}(\mathrm{ODe}=$ decyloxide $)$, prepared according to a previously developed procedure [23]. In this case the thermal treatment was made in situ before the WGSR tests. The materials were treated under $\mathrm{H}_{2}(10 \%)$ in Argon (total flow $49 \mathrm{~mL} \mathrm{~min}{ }^{-1}$ ) for 1 hour at $250{ }^{\circ} \mathrm{C}$.

The relative percentage of $\mathrm{Pd@MO} 2(\mathrm{M}=\mathrm{Ti}$ or $\mathrm{Ce})$ was calculated based on TGA data. In particular, the Fe:CNT ratio was first evaluated and it was assumed that such ratio remains constant after hybridization with the Pd@MO 2 . After observing the CNT \% in the final hybrid, the Fe proportion could therefore be calculated and the amount of $\mathrm{Pd@MO}$ evaluated by difference between the total inorganic residue and the Fe.

\subsection{Characterization}

Thermogravimetric analyses (TGA) of approximately $1 \mathrm{mg}$ of each compound is recorded on a TGA Q500 (TA Instruments) under air, by equilibrating at $100{ }^{\circ} \mathrm{C}$ for $20 \mathrm{~min}$, and following a ramp of $10^{\circ} \mathrm{C} \mathrm{min}^{-1} \mathrm{up}$ to $800{ }^{\circ} \mathrm{C}$. Raman spectra are recorded with an inVia Renishaw microspectrometer equipped with $\mathrm{Nd}$ :YAG laser at $532 \mathrm{~nm}$. To avoid sample damage or laser-induced heating/crystallization of the materials, the incident power was kept at $1 \%$ (full power of the laser is $100 \mathrm{~mW}$. Powders are dispersed in $\mathrm{EtOH}$, drop-cast onto a quartz slide and the solvent evaporated; at least 5 spectra per sample are recorded on different areas of the sample in order to check the uniformity of the materials. TEM was performed using a JEOL 2100F transmission electron microscope operating at $200 \mathrm{kV}$ (field emission electron gun source, information limit $0.19 \mathrm{~nm}$ ). TEM samples were prepared by casting several drops of 
methanolic suspensions onto a copper mesh or lacey carbon TEM grid and dried under ambient conditions. STEM images were acquired using a JEOL digital STEM system. EDX spectroscopy was performed using an Oxford Instruments XMax 80 T silicon drift detector with INCA Energy 250 Microanalysis system. Additional TEM images were acquired on a were performed on a TEM Philips EM208, using an acceleration voltage of $100 \mathrm{kV}$.

Textural properties have been analyzed by $\mathrm{N}_{2}$ physisorption at the liquid nitrogen temperature using a Micromeritics ASAP 2020 analyzer. Prior to the analysis, the samples have been degassed at $120{ }^{\circ} \mathrm{C}$ for $12 \mathrm{~h}$. The surface area has been calculated adopting the Brunauer-Emmett-Teller (BET) model while the pore size distribution has been assessed following the Barrett-Joyner-Halenda (BJH) Analysis.

$\mathrm{CO}$ chemisorption experiments were carried out on $\mathrm{CeO}_{2}$-based materials using a Micromeritics ASAP 2020C. Typically, $150 \mathrm{mg}$ of the fresh material were loaded in a U-shaped reactor and reduced by flowing $\mathrm{H}_{2}(5 \%) / \operatorname{Ar}\left(40 \mathrm{~mL} \mathrm{~min}{ }^{-1}\right)$ at $120^{\circ} \mathrm{C}$ for $1 \mathrm{~h}$. The adsorbed hydrogen is then removed by evacuation at $250{ }^{\circ} \mathrm{C}$ for $6 \mathrm{~h}$. Chemisorption analysis was performed firstly at $35^{\circ} \mathrm{C}$ dosing $\mathrm{CO}(2-20$ torr). A low pressure range have been employed to avoid carbonate formation on $\mathrm{CeO}_{2}$-based materials. The contribution of physisorption was subtracted by the double-isotherm method. $\mathrm{A} C \mathrm{CO} / \mathrm{Pd}$ stoichiometry of 1:1 has been employed to calculate the accessible fraction of the metal.

The effective composition of the prepared materials was determined by ICP-AES analysis after the optimization of an adequate dissolution procedure. To do that, $20 \mathrm{mg}$ the calcined samples were firstly heated in air at $600{ }^{\circ} \mathrm{C}$ within an $\mathrm{Al}_{2} \mathrm{O}_{3}$ crucible to burn the carbon-based structure. After that, the remaining powder was dissolved by a triacid attack $\left(0.5 \mathrm{~mL} \mathrm{HF} 48 \%+0.5 \mathrm{~mL} \mathrm{HNO}_{3} 65 \%+0.1 \mathrm{~mL} \mathrm{HClO}_{4}\right.$ $70 \%)$ at $80{ }^{\circ} \mathrm{C}$ for $2 \mathrm{~h}$ [24]. After dilution to $25.00 \mathrm{~mL}$ with water, the $\mathrm{Fe}, \mathrm{Ce}, \mathrm{Ti}$ and $\mathrm{Pd}$ concentrations were determined by ICP-AES analysis measuring the emission at 238.204, 413.764, 334.940 and 340.458 $\mathrm{nm}$, respectively. Adequate calibration lines have been built using at least 5 points in the concentration range of the analysed metal, obtaining a $R^{2}>0.9995$.

X-Ray Absorption experiments were performed at the SAMBA beamline [25] of Synchrotron SOLEIL (France) with a Si (220) double crystal monochromator. The monochromator was kept fully tuned and harmonics were rejected by a pair of Pd-coated, Si mirrors. Spectra were measured in fluorescence mode using a 35-element Ge detector. One ionization chamber was used as the baseline monitor and two other chambers were used to continuously check the stability of the energy scale by placing a Fe foil as reference. The experiments were conducted loading $50 \mathrm{mg}$ of the as-prepared Fe@CNT/Pd@TiO 2 
material within the quartz capillary (thickness $=10 \mu \mathrm{m}$ ) between two quartz wool plugs. $\mathrm{H}_{2}(5 \%) / \mathrm{N}_{2}$ was flowed on the sample $\left(30 \mathrm{~mL} \mathrm{~min}^{-1}\right)$ and the temperature was raised with a ramp of $5^{\circ} \mathrm{C} \mathrm{min}^{-1}$ using a gas blower. XANES/EXAFS spectra were recorded at the Fe $\mathrm{K}$ edge $(7112 \mathrm{eV})$ in a continuous mode (1 full spectra every $2 \mathrm{~min})$.

\subsection{Photocatalytic tests}

The materials were tested as photocatalyst for hydrogen production by photoreforming of an ethanol/water mixture (1/1 by volume) under two different light sources. A UV $125 \mathrm{~W}$ medium pressure Hg lamp (model UV13F, Helios Italquartz, Italy) was used to illuminate a Pyrex photoreactor while a Teflon-lined photoreactor was illuminated with a Lot-Oriel Solar Simulator equipped with a $150 \mathrm{~W}$ Xe lamp and an Atmospheric Edge Filter with a cut-off at $300 \mathrm{~nm}$. In a typical catalytic test, $10 \mathrm{mg}$ of the calcined material was first suspended in the photoreactor by sonication for 10 minutes in $60 \mathrm{~mL}$ of ethanol solution and subsequently purged from air with Ar flow of $15 \mathrm{~mL} \mathrm{~min}^{-1}$ for 40 minutes and thermostated at $20^{\circ} \mathrm{C}$. During the purge and the catalytic test the materials were magnetically stirred. The on-line detection of volatile products was carried out using a Gas Chromatograph equipped with two analytical lines and a 10 way-two loops injection valve was employed for injection during on-line analysis of the gaseous products. In the former apparatus an Agilent 6890N Gas Chromatograph equipped with a MolSieve $5 \AA$ (Restek, $30 \mathrm{~m}$ x $0.53 \mathrm{~mm}$ ID) column followed by a Thermal Conductivity Detector (TCD) was used for gaseous products quantification using Ar as carrier and a PoraPlot $Q$ (Agilent, $30 \mathrm{~m} \times 0.53 \mathrm{~mm}$ ID, $40 \mu \mathrm{m}$ film) column using Ar as a carrier followed by a methanator and a Flame Ionization Detector (FID) was employed for the detection of the volatile organic compounds. In the latter apparatus an Agilent 7890A Gas Chromatograph equipped with a Carboxen 1010 PLOT (Supelco, $30 \mathrm{~m} \times 0.53 \mathrm{~mm}$ ID, $30 \mu \mathrm{m}$ film) column followed by a Thermal Conductivity Detector (TCD) was used for gaseous products quantification using Ar as carrier and a DB-225 ms column (J\&W, $60 \mathrm{~m} \times$ $0.32 \mathrm{~mm}$ ID, $20 \mu \mathrm{m}$ film) using He as carrier followed by a mass spectrometer (MS) HP 5975C for the detection of the volatile organic compounds.

\subsection{WGSR tests}

WGSR rates were measured in a tubular reactor at atmospheric pressure, using partial pressures of 7.6 Torr and 38 Torr each for $\mathrm{CO}$ and $\mathrm{H}_{2} \mathrm{O}$ respectively. The gas hourly space velocity (GHSV) was set at $100000 \mathrm{~h}^{-1}$ using a total flow rate of $48.5 \mathrm{~mL} \mathrm{~min}^{-1}$ and $29 \mathrm{mg}$ of the powdered catalyst. Water was introduced to the reactor by means of a Gas-Tight syringe, controlled by an infusion pump, while the 
partial pressures of $\mathrm{CO}$ and $\mathrm{Ar}$ were adjusted using mass flow controllers. The composition of the effluent from the reactor was monitored on-line using an Agilent 7890 gas chromatograph equipped with two analytical lines. A TCD detector, connected to a MolSieve $5 \AA$ column using Ar as carrier, was used to monitor $\mathrm{H}_{2}$ concentration while a FID detector, connected to a Select Permanent Gases $/ \mathrm{CO}_{2}$ column using $\mathrm{He}$ as carrier and a methanator, was used to monitor $\mathrm{CO}, \mathrm{CH}_{4}$ and $\mathrm{CO}_{2}$.

\section{Results and discussion}

Our investigations started with the preparation of magnetic Fe@CNTs, enriched with $\alpha-\mathrm{Fe}$ and $\mathrm{Fe}_{3} \mathrm{C}$, following a procedure previously developed by some of us [14]. Access to the hierarchical hybrids was attained through adaptation of a reported protocol [17]: the Fe@CNTs are covalently functionalized with benzoic acid units [18], which improve dispersibility in the polar liquid medium and serve as anchoring points for attaching the pre-assembled inorganic Pd-MUA@M(OR $)_{4}$ precursor $(\mathrm{MUA}=$ mercaptoundecanoic acid, $\mathrm{M}=\mathrm{Ti}$ or $\mathrm{Ce}, \mathrm{R}=-$ Butyl or -Decyl). Controlled hydrolysis and magnetic separation afford the CNT-enriched nanohybrid (Fe@CNTs/Pd@MO comparison, the corresponding catalyst separated by filtration was also prepared (Fe@CNTs/Pd@MO $2^{-}$ filt). Two different metal oxides, each for a specific catalytic purpose, were chosen: $\mathrm{TiO}_{2}$ as photoactive material in the photocatalytic hydrogen evolution and $\mathrm{CeO}_{2}$ as an active support in the WGSR. 


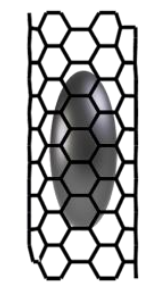

A
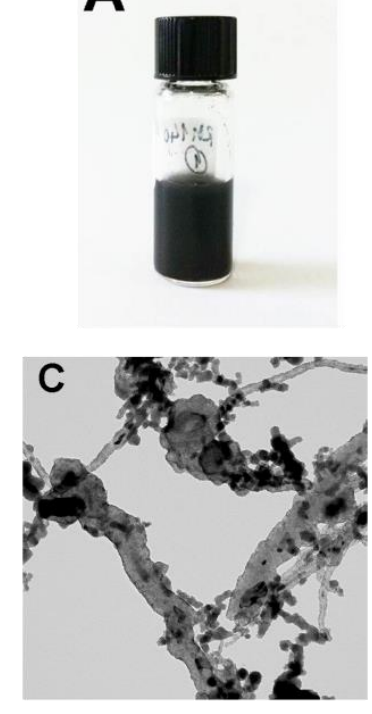

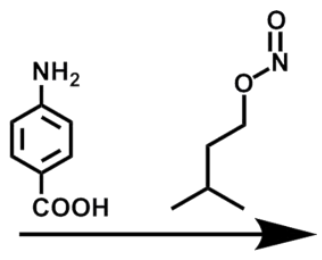

NMP, $90^{\circ} \mathrm{C}$
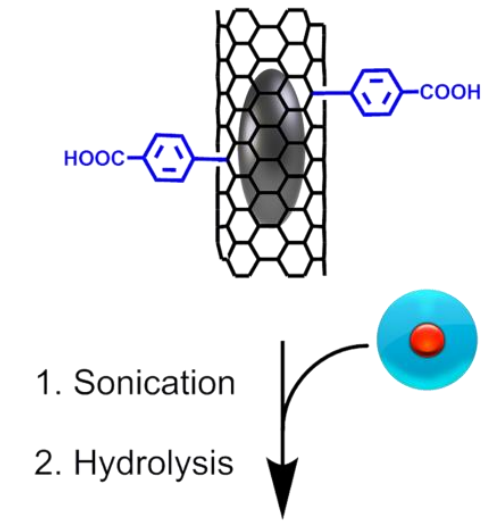

B
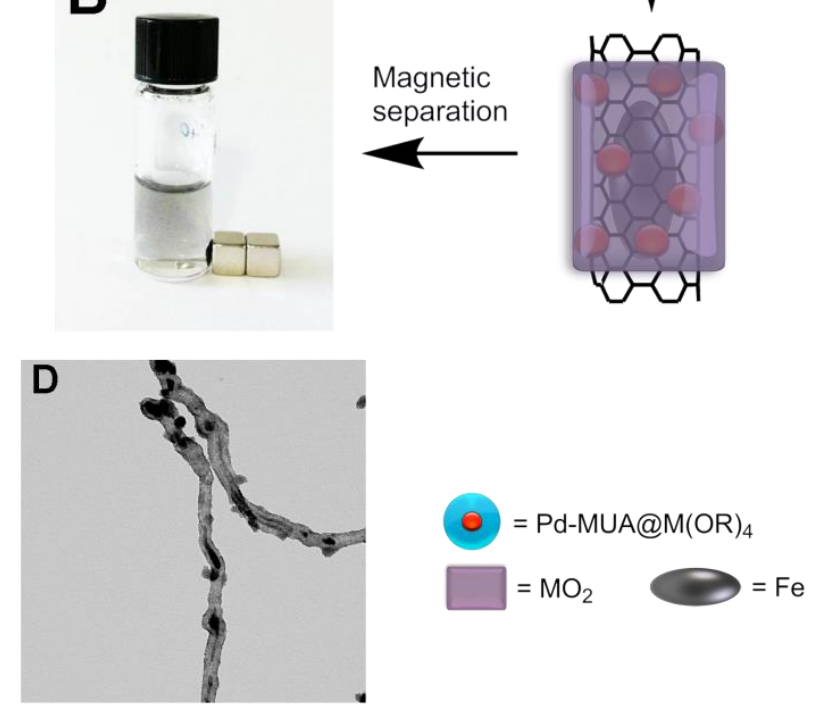

Scheme 1: Graphical general scheme of the synthesis of Fe@CNTs/Pd@MO prepared hybrid and C) corresponding representative TEM image relative to the $\mathrm{TiO}_{2}$-based material. B) Separation of the magnetic solid with a magnet leaving the liquid phase containing non-magnetic impurities and (D) representative TEM image of the separated solid.

As a final treatment, a controlled thermal treatment of the as prepared nanohybrid allows stabilization of the inorganic component by crystallization of the oxide phase, and good accessibility to the Pd nanoparticles upon removal of the organic moieties used as protecting agents. In the case of the present materials, the thermal treatment has been performed under reducing conditions in flowing $\mathrm{H}_{2} / \operatorname{Ar}(1: 10)$ instead of the previously employed oxidizing conditions (static air) [8]. XANES/EXAFS analysis of the Fe component (Fig. 1) revealed that, in the fresh materials, mainly $\mathrm{Fe}_{3} \mathrm{C}$ is present, as also observed in the 
Fe@CNTs precursor by TEM and SAED inspection (Fig. S3). This is in agreement with our previous studies $[17,19]$ and suggests that the coating procedure with the inorganic oxides did not affect the encapsulated magnetic component. Increasing the temperature during $\mathrm{H}_{2}$ treatment, some subtle modifications appear in the XANES spectra, suggesting that $\mathrm{Fe}_{3} \mathrm{C}$ is partially converted into $\mathrm{Fe}$ (see Fig. S1, part A). The contribution of the two components $\left(\mathrm{Fe}_{3} \mathrm{C}\right.$ and $\left.\mathrm{Fe}\right)$ has been calculated by linear combination fitting of the XANES spectra acquired at different temperatures. Inset of Fig. 1 shows how the relative content of metallic Fe progressively increases at the reduction temperature increases up to $48 \%$ after reductive treatment at $450{ }^{\circ} \mathrm{C}$ (Fig. S1). Notably, no formation of $\mathrm{FeO}_{\mathrm{x}}$ was also observed during thermal treatment under $\mathrm{H}_{2} / \mathrm{Ar}$ atmosphere. On the other hand, the formation of $\mathrm{FeO}_{\mathrm{x}}$ was observed when an oxidative treatment was applied at the same temperature $\left(450^{\circ} \mathrm{C}\right)$ : the fitting of the XANES pattern (Fig. S2) revealed that the sample contains around $55 \mathrm{wt} \%$ of metallic Fe and $45 \%$ of $\gamma$ $\mathrm{Fe}_{2} \mathrm{O}_{3}$.

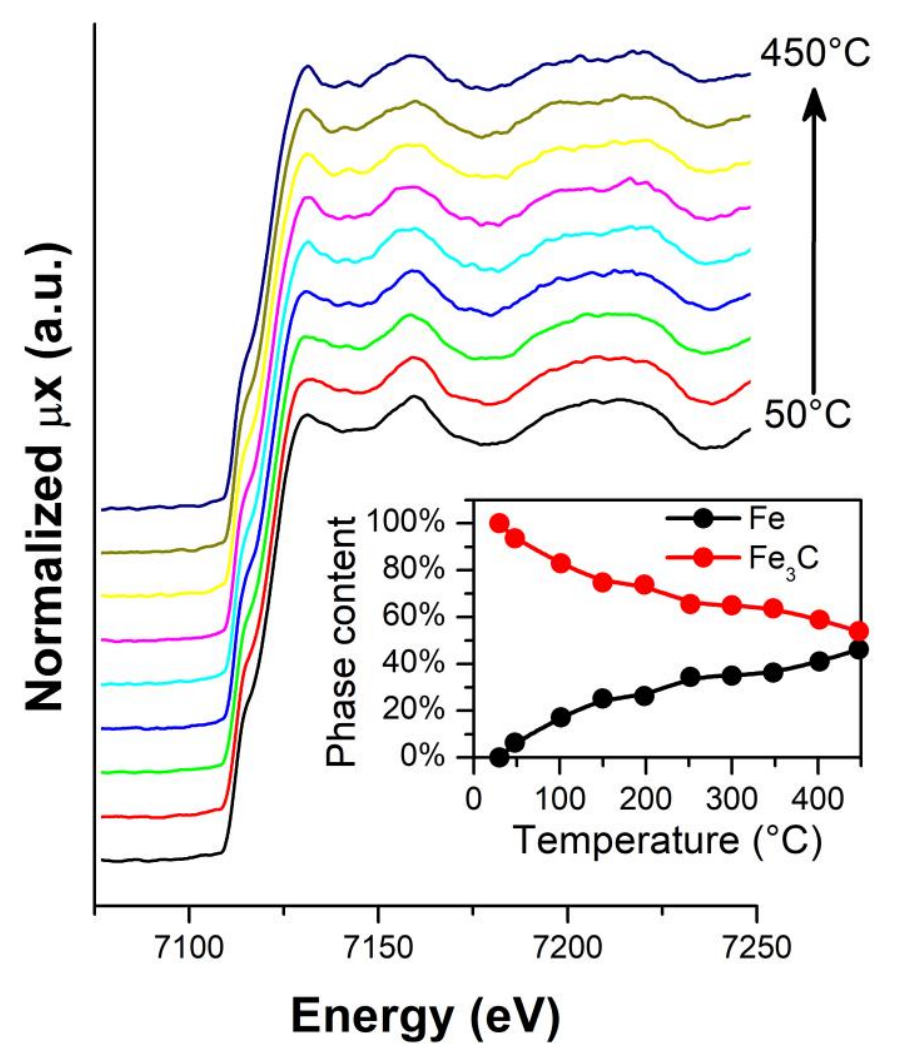


Fig. 1: XANES spectra at different temperatures recorded at the Fe $\mathrm{K}$ edge during treatment in $\mathrm{H}_{2}(5 \%) / \mathrm{N}_{2}$ of the Fe@CNTs/Pd@TiO2-magn material. Inset: evolution of $\mathrm{Fe}_{3} \mathrm{C}$ and Fe contents as derived from linear combination fitting of the XANES spectra.

\subsection{Fe@CNTs/Pd@TiO for photocatalytic $\mathrm{H}_{2}$ evolution.}

Prior to the catalytic tests, the material was subjected to a thermal treatment under $\mathrm{H}_{2} / \operatorname{Ar}(1: 10)$ flow at $450{ }^{\circ} \mathrm{C}$ to remove the organic ligands and crystallize the metal oxide. In this case, the reducing treatment is beneficial for titania-based material as it also causes an enhancement of the photocatalytic activity due to the formation of $\mathrm{Ti}^{3+}$ centers associated with $\mathrm{O}$ vacancies [26-28]. Unfortunately, the quantification of the contribution of $\mathrm{Ti}^{3+}$ centers to catalysis could not be directly carried out for the present material. In fact, the corresponding thermal treatment of the magnetic catalyst in absence of $\mathrm{H}_{2}$ (in order not to reduce $\mathrm{Ti}^{4+}$ species) resulted in concomitant oxidation of Fe, which compromises the catalytic activity. Thermogravimetric analysis (TGA) confirms that the two methods of isolation of the final material (magnetic separation vs filtration) lead to different CNT:inorganic weight ratios (0.85 and 0.24 for Fe@CNTs/Pd@TiO 2 -magn and Fe@CNTs/Pd@TiO 2 -filt, respectively) implying the presence of excess and free standing $\mathrm{Pd} @ \mathrm{TiO}_{2}$ in the latter (Fig. 2A). From the analysis it can also be appreciated that in the as prepared material there is a small percentage of organic matter (combustion temperature $250{ }^{\circ} \mathrm{C}$ ), related to the organic ligands of the metal precursors, which is totally removed during the thermal treatment. Furthermore, the TGA of the $f$-Fe@CNTs shows that the residual Fe derivative accounts for about $23 \%$ (Fig. 2A).

Chemical analysis of the thermally treated materials performed by ICP-AES revealed the real composition of the obtained materials. The Fe@CNTs/Pd@TiO -filt sample composition is (by weight percent) Pd 1.01\%, $\mathrm{TiO}_{2}$ 68.5\% and Fe 7.23\%, while the Fe@CNTs/Pd@TiO - -magn sample contains Pd $0.57 \%, \mathrm{TiO}_{2} 34.9 \%$ and $\mathrm{Fe} 15.1 \%$. From these data, the carbon content was calculated as $23.3 \%$ and 49.4\% for the filtered and magnetically separated materials, respectively. These values are larger than the weight loss observed during TGA (19.5\% and 43.1\% for Fe@CNTs/Pd@TiO Fe@CNTs/Pd@TiO 2 -filt, respectively, Fig 2A). This is due to the fact that Fe component is oxidized to $\mathrm{Fe}_{2} \mathrm{O}_{3}$ during TGA, accounting for the weight loss due to carbon burning and the weight gain due to $\mathrm{Fe}$ oxidation. However, the difference in CNT:inorganic ratio is not substantially altered by calculations based on ICP-AES analysis (0.98 for Fe@CNTs/Pd@TiO 2 -magn vs 0.30 for Fe@CNTs/Pd@TiO 2 -filt). 

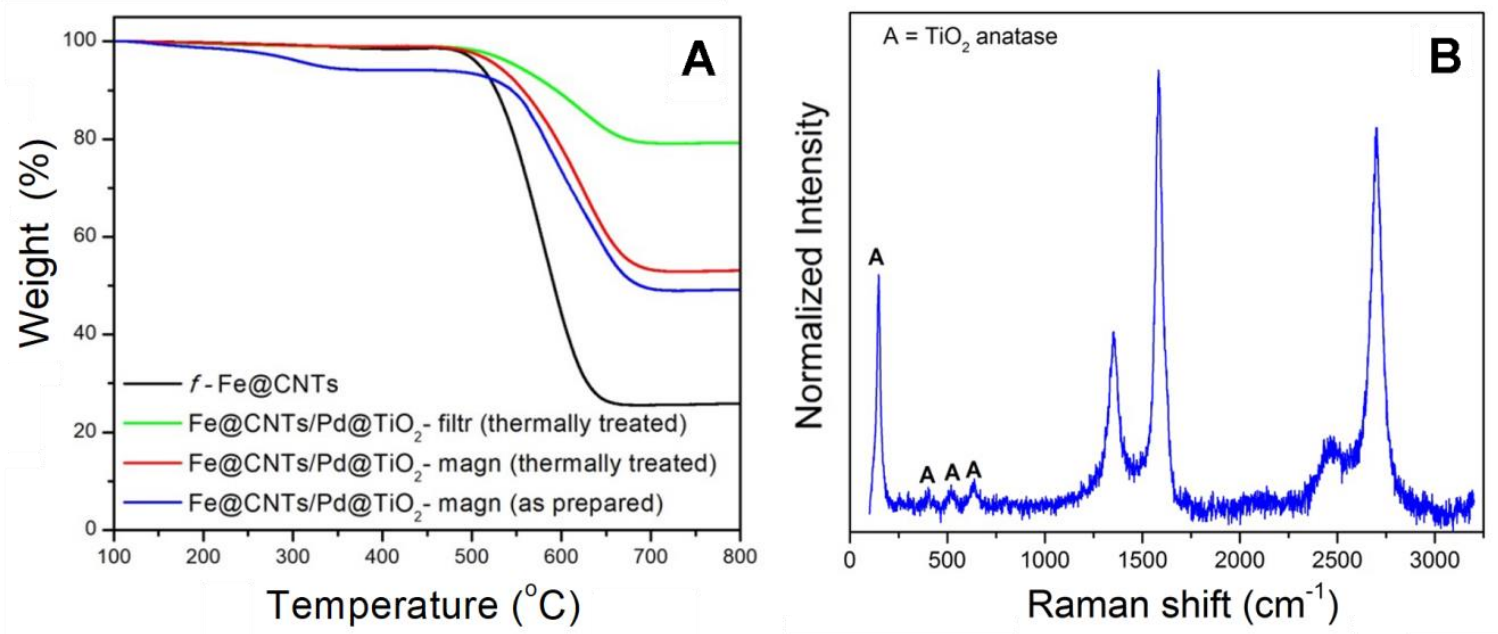

Fig. 2. Thermogravimetric analysis (TGA) of the $\mathrm{TiO}_{2}$-based hybrids (A) and Raman spectrum of the thermally treated Fe@CNTs/Pd@TiO2-magn (B)

Raman analysis confirms that the CNT scaffold is retained after the calcination step, with the presence of the typical $D$ and $G$ bands respectively at $\sim 1300 \mathrm{~cm}^{-1}$ and $\sim 1590 \mathrm{~cm}^{-1}$, together with the formation of $\mathrm{TiO}_{2}$ anatase (bands at $146 \mathrm{~cm}^{-1}, 198 \mathrm{~cm}^{-1}, 395 \mathrm{~cm}^{-1}, 513 \mathrm{~cm}^{-1}$ and $639 \mathrm{~cm}^{-1}$ ) [29, 30]. However, no bands associated to Fe oxides are found, further confirming the perfect isolation of the iron phase in the carbon nanotube cavity (Fig. 2B).

The homogeneous coverage of $\mathrm{CNTs}$ by $\mathrm{TiO}_{2}$ incorporating $\mathrm{Pd}$ nanoparticles and the close contact between the CNT and inorganic components become evident through TEM analysis (Fig. 3A), which also indicates that encapsulation of Fe within the CNTs is retained after the synthetic procedure (Fig. 3B-C). Moreover, TEM inspection rules out any significant free-standing inorganic phase for Fe@CNTs/Pd@TiO 2 -magn, while its presence is clearly observed for the Fe@CNTs/Pd@TiO (Fig. S4). In addition, TEM analysis of the discarded liquid phase after magnetic purification of Fe@CNTs/Pd@TiO 2 -magn provides evidence of the presence of large $\mathrm{TiO}_{2}$ aggregates (Fig. S5). EDX mapping confirms the presence and almost colocalization of $\mathrm{C}, \mathrm{Ti}$ and $\mathrm{O}$, with Fe encapsulated within CNTs (Fig. 3D-E). Fast Fourier Transform (FFT) additionally confirms that the $\mathrm{TiO}_{2}$ is mostly in the anatase phase, contaminated with a tiny portion of rutile (Fig. S6). The higher element-dependent contrast of the HAADF images permits to clearly reveal the presence of the Pd nanoparticles (they appear as white compact dots due to the fact that Pd has the highest atomic number in this material) with a size distribution of $3.5 \pm 0.6 \mathrm{~nm}$ (Fig. $3 \mathrm{C}$ and inset), also confirmed by the EDX analysis (Fig. S7). 

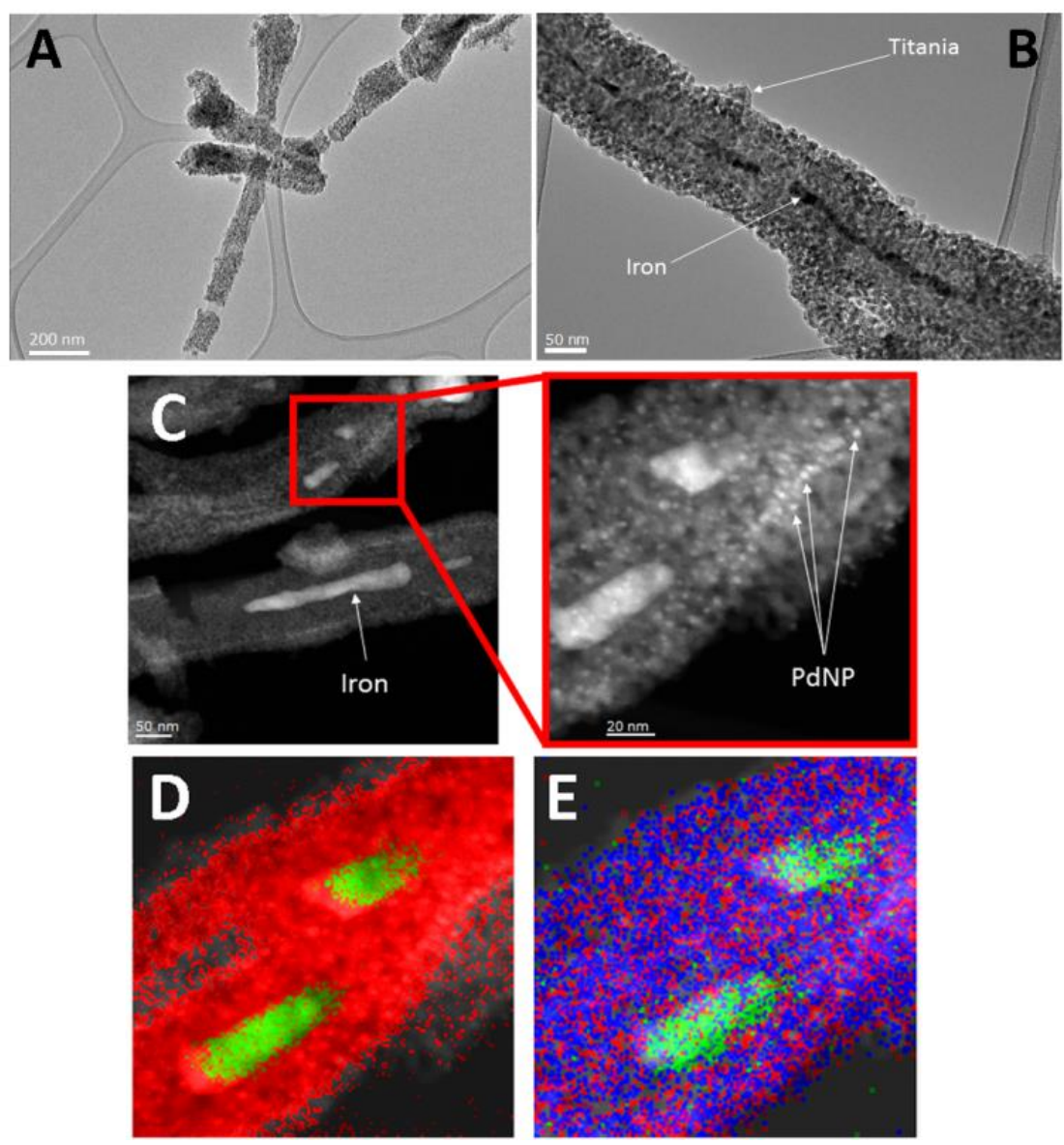

Fig. 3. A) low magnification and B) high magnification HRTEM and C) HAADF-STEM images of sample Fe@CNTs/Pd@TiO2-magn thermally treated (inset of C: magnification showing the individual Pd NPs); D) EDX mapping showing carbon (red) and iron (green); E) EDX mapping showing titanium (blue), oxygen (red) and iron (green).

It is interesting to note how the high temperature treatment does not cause agglomeration of Pd NPs into large aggregates, thanks to the protection ensured by the $\mathrm{TiO}_{2}$ shell. A complete EDX mapping of all the elements together with a high resolution mapping of $\mathrm{Pd}$ and $\mathrm{C}$ reveal that the $\mathrm{Pd}$ nanoparticles are well dispersed along the CNT (Fig 4). 

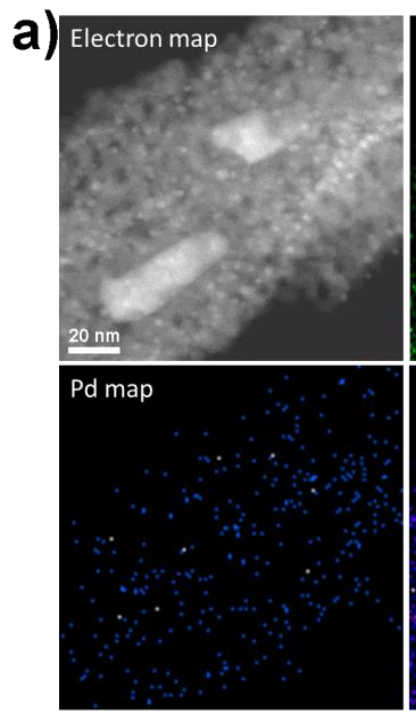
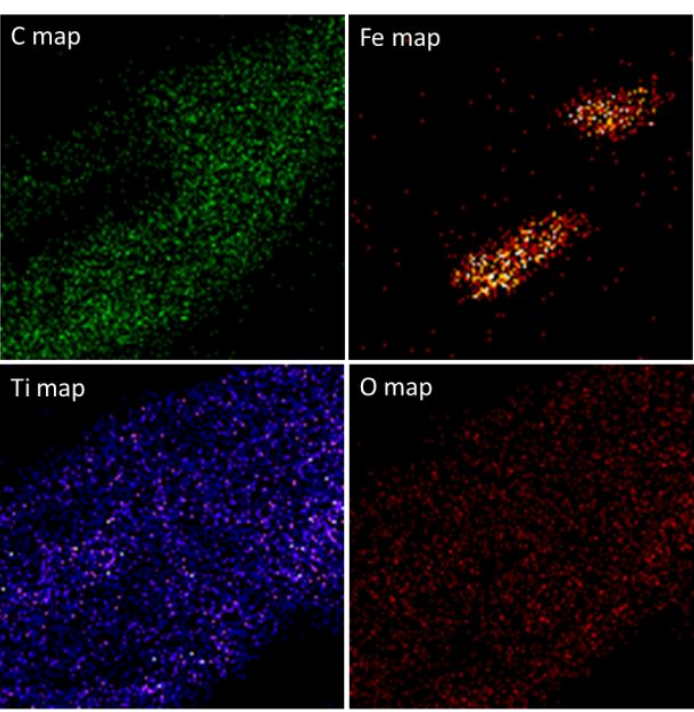

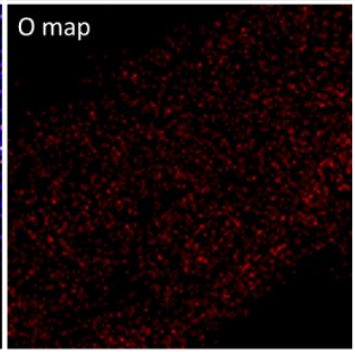

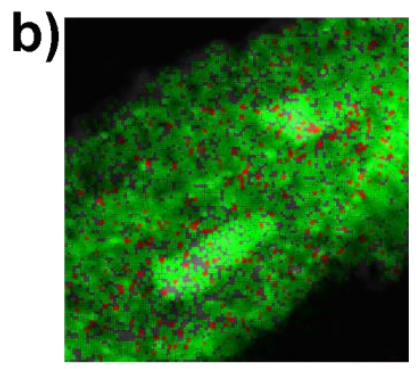

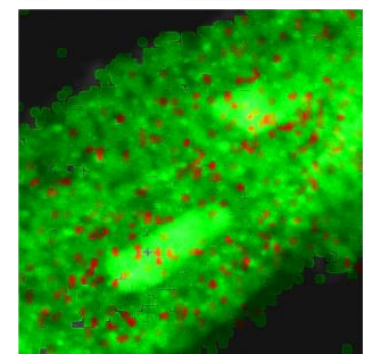

Fig. 4. a) HRTEM (top left) and EDX mapping of all the elements of Fe@CNTs/Pd@TiO2-magn and b) Highresolution (top) and low-resolution (bottom) mapping of Pd nanoparticles (red) on carbon (green).

The textural properties of both thermally-treated Fe@CNTs/Pd@TiO - -magn and Fe@CNTs/Pd@TiO ${ }_{2}$-filt, as well as those of the $f$-Fe@CNTs, were investigated by $\mathrm{N}_{2}$ physisorption. All samples exhibit a type IV isotherm indicating extended mesoporous texture (Fig. S8). The main results from $\mathrm{N}_{2}$ physisorption are summarized in Table 1. Despite the quite low surface areas of Fe@CNTs/Pd@TiO ${ }_{2}$-magn and Fe@CNTs/Pd@TiO 2 -filt (30 and $28 \mathrm{~m}^{2} \mathrm{~g}^{-1}$ respectively), the differences in the texture of the various materials are well evident. In particular, the hybrid materials show higher surface area (surface area of $f$ Fe@CNTs $\left.17 \mathrm{~m}^{2} \mathrm{~g}^{-1}\right)$ but lower pore volume than the $f$-Fe@CNT $\left(0.074\right.$ and $0.050 \mathrm{~m}^{3} \mathrm{~g}^{-1}$ for the two hybrids vs $0.111 \mathrm{~m}^{3} \mathrm{~g}^{-1}$ for the $f$-Fe@CNTs, see Table 1). This is clearly related with the growth of the mesoporous $\mathrm{Pd@} \mathrm{TiO}_{2}$ layer, which is characterized by the presence of mesopores around $4 \mathrm{~nm}$ in the Barrett-Joyner-Halenda (BJH) pore distributions (Fig. S9).

\section{Table 1}

Summary of results from analysis of the $\mathrm{N}_{2}$ physisoprtion isotherms of the $\mathrm{TiO}_{2}$-based samples.

\begin{tabular}{|c|c|c|c|}
\hline Sample & $f$-Fe@CNTs & $\begin{array}{c}\text { Fe@CNTs/Pd@TiO }{ }_{2} \text {-magn } \\
\text { (thermally treated) }\end{array}$ & $\begin{array}{c}\text { Fe@CNTs/Pd@TiO }{ }_{2} \text { filtr } \\
\text { (thermally treated) }\end{array}$ \\
\hline Type of isotherm $^{\text {a }}$ & II & IV & IV \\
\hline
\end{tabular}




\begin{tabular}{|c|c|c|c|}
\hline Specific Surface Area $\left(\mathrm{m}^{\mathbf{2}} / \mathbf{g}\right)^{\mathbf{b}}$ & 17 & 30 & 28 \\
\hline Cumulative Pore Volume $(\mathrm{mL} / \mathrm{g})^{\mathrm{c}}$ & 0.111 & 0.074 & 0.050 \\
\hline$D_{\max }(\mathrm{nm})^{d}$ & 77 & $4.0 / 113$ & $3.7 / 95$ \\
\hline
\end{tabular}

${ }^{\text {a }}$ accordingly to IUPAC recommendation [31].

${ }^{\mathrm{b}}$ Calculated using the BET equation.

${ }^{\mathrm{c}}$ Calculated from the BJH analysis of the desorption branch of the $\mathrm{N}_{2}$ physisorption isotherms.

${ }^{\mathrm{d}}$ Maximum of the pore size distribution obtained applying the BJH analysis to the adsorption branch of the $\mathrm{N}_{2}$ physisorption isotherms, reported in Fig. S9.

The photocatalytic performance of the two thermally-treated catalysts was evaluated for the $\mathrm{H}_{2}$ evolution from EtOH/ $\mathrm{H}_{2} \mathrm{O}$ solution under UV-vis irradiation. A comparison between the activities of the two materials undeniably displays a considerably superior performance of the Fe@CNTs/Pd@TiO -magn $^{-}$ sample, reaching a stable $\mathrm{H}_{2}$ productivity amounting to $1092 \mathrm{mmol}_{\text {Pd@TiO2 }}{ }^{-1}$ after 20 hours of

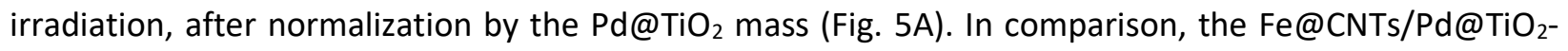
filt performance is about $46 \%$ lower $\left(510 \mathrm{mmol}_{\mathrm{Pd}_{\mathrm{PTiO}}}{ }^{-1}\right)$. The stability of both materials is pronounced, with steady $\mathrm{H}_{2}$ production for at least 20 hours of catalysis. The beneficial effect of the thermal treatment was also proven by comparing the activities of the as-prepared sample with those observed from the thermally treated material (Fig. S10), with the latter displaying an almost five-fold higher activity than the former. This effect is well known and is related to the crystallinity of the titania shell which bears an enhanced light absorption characteristics with lower band gaps than the amorphous $\mathrm{TiO}_{2}$

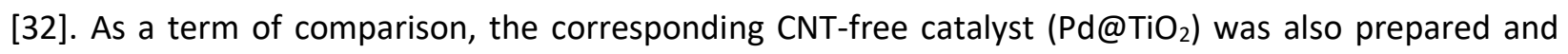
displayed even lower activity, indicating the benefits of including CNT in the catalyst formulation. A comparison with Fe-free analogues cannot be done as it is misleading, due to the fact that it is not possible to prepare uniformly covered CNTs without the presence of additional isolated $\mathrm{Pd@MO} 2$ component (whose percentage content is not measurable), making the direct comparison affected by a considerable bias.

The magnetic properties of the nanotubes can also be exploited for the post catalytic recovery of the catalyst. Recycling tests based on magnetic separation from the liquid phase after 3 cycles of simulated solar irradiation shows no significant decrease of activity. This implies that the material preserves its 
structural and catalytic characteristics and that the magnetic recovery method is simple, robust and reproducible (Figure 5B).
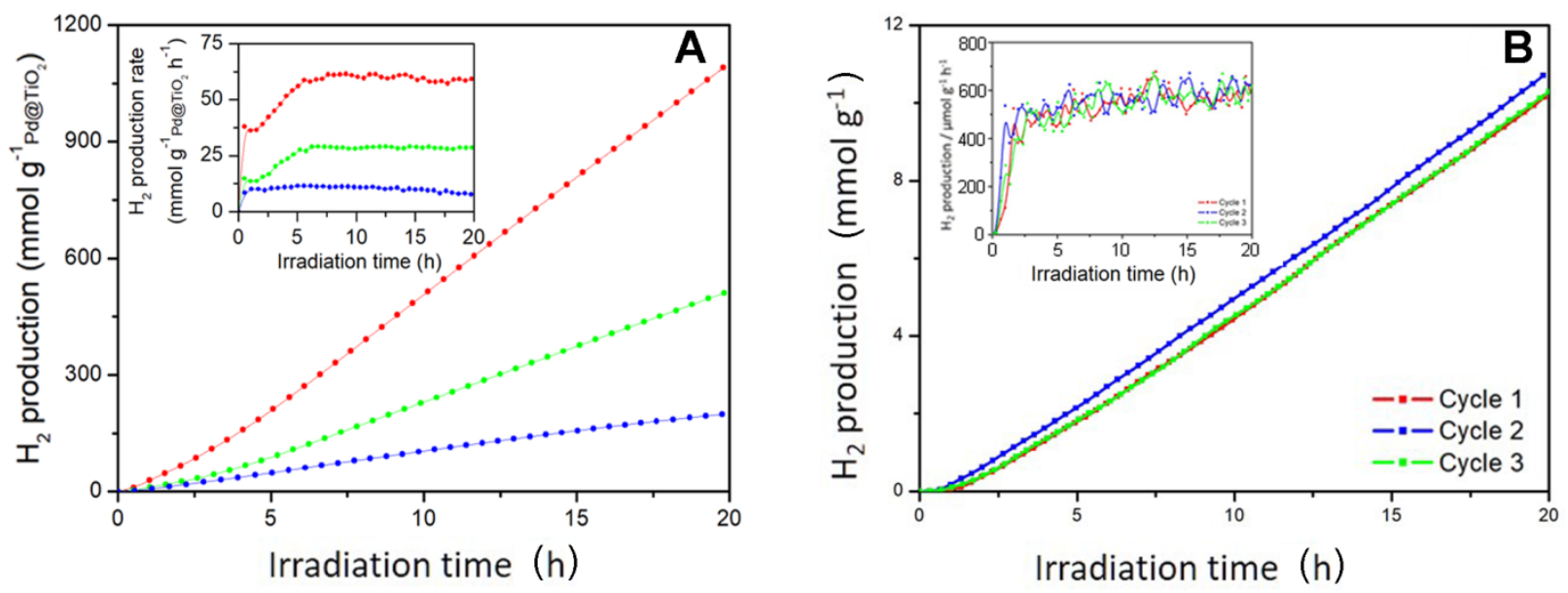

Fig. 5. A) Photocatalytic $\mathrm{H}_{2}$ production over 20 hours under UV irradiation by the thermally treated Fe@CNTs/Pd@TiO2-magn (red), Fe@CNTs/Pd@TiO2-filt (green) and CNT-free Pd@TiOz (blue) normalized by the Pd@TiO2 content. Inset: $\mathrm{H}_{2}$ evolution rates. B) Photocatalytic $\mathrm{H}_{2}$ production under simulated solar light by the thermally treated Fe@CNTs/Pd@TiO 2 -magn over three cycles after magnetic recovery of the catalysts. Inset: $\mathrm{H}_{2}$ evolution rates.

\subsection{Fe@CNTs/Pd@CeO for WGSR}

As a further proof of the key importance of the homogeneity of the CNT hybrids, the synthesis and performance towards the WGSR ( $\left.\mathrm{CO}+\mathrm{H}_{2} \mathrm{O} \leftrightarrow \mathrm{H}_{2}+\mathrm{CO}_{2}\right)$ of Fe@CNTs/Pd@CeO $\mathrm{O}_{2}$ was evaluated. In this case, $\mathrm{CeO}_{2}$ was chosen as a metal oxide layer given its well-known promoting effect for catalytic WGSR [33]. The advantages of integrating CNTs with $\mathrm{Pd@} \mathrm{CeO}_{2}$ in the WGSR have been recently proved [34], and in particular it has been highlighted that an optimum $\mathrm{CeO}_{2} / \mathrm{CNT}$ ratio, that causes the most homogeneous coverage of the carbon scaffold, also reflects in a superior catalytic activity and stability [8].

The as prepared hybrids were first compared based on their composition, obtained from TGA and ICPAES data. TGA analysis in air flow of the as prepared materials show a 2-step weight loss, the first one (around $200{ }^{\circ} \mathrm{C}$ ) related with removal of organic capping agents and the second one (from 400 to $600{ }^{\circ} \mathrm{C}$ ) due to combustion of CNT scaffold (Fig 6A). ICP-AES analysis revealed that the composition of the 
thermally treated materials are Pd $1.31 \%, \mathrm{CeO}_{2} 69.5 \%$, Fe 7.30 and C $21.9 \%$ for Fe@CNTs/Pd@CeO $2^{-}$ magn and $\mathrm{Pd}$ 1.65\%, $\mathrm{CeO}_{2}$ 84.8\%, Fe 3.75\% and C 9.80\% for Fe@CNTs/Pd@CeO 2 -filt. Also in this case, the real $\mathrm{C}$ content is higher than the weight loss detected by TGA in air in the range $400-600{ }^{\circ} \mathrm{C}$ (19.05\% and $8.15 \%$ for magnetically separated and filtered samples, respectively), since Fe oxidation results in a gain in the weight of the sample that superimposes to the weight loss due to CNT combustion. As in the previous case, a higher CNT:inorganic weight ratio $(0.28$ vs 0.11$)$ for the magnetically purified material has been observed (based on the more accurate ICP-AES analysis).
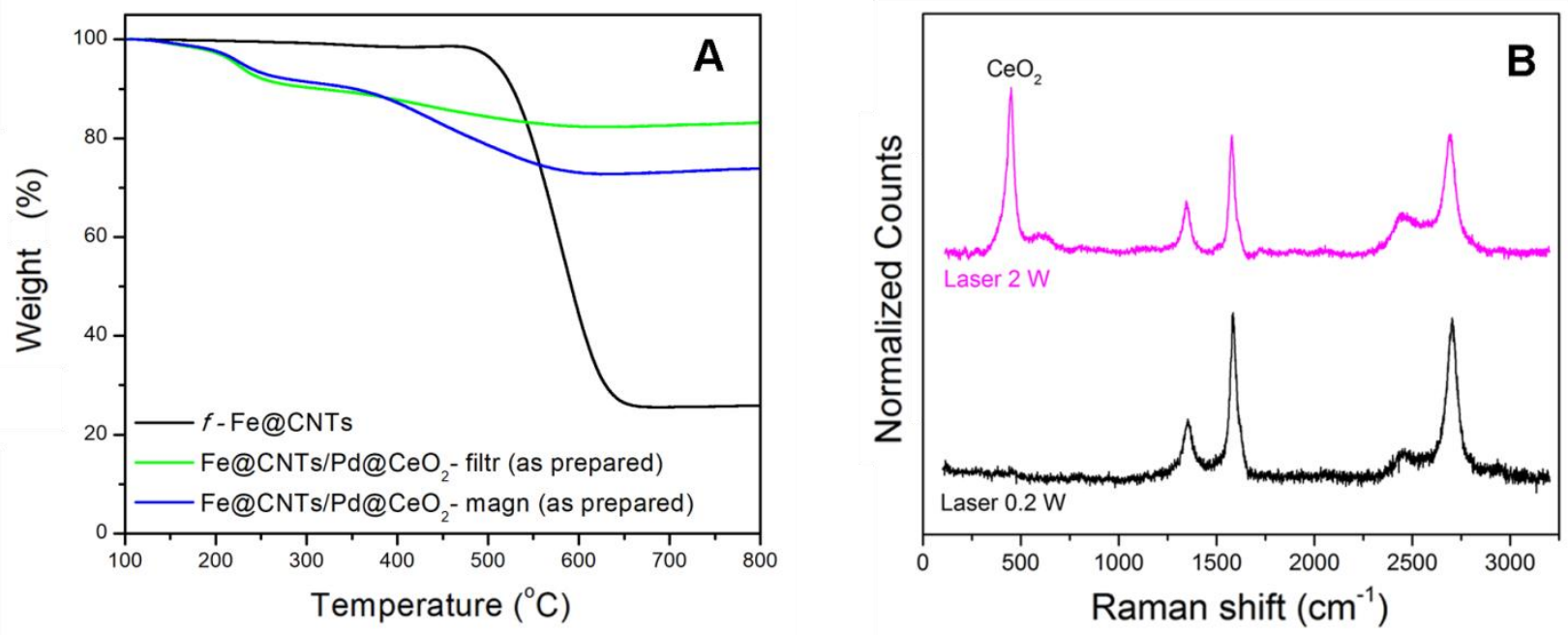

Fig. 6. A) Thermogravimetric analysis (TGA) and B) Raman spectra of the $\mathrm{CeO}_{2}$-based hybrids.

Raman analysis shows no signal relative to the $\mathrm{CeO}_{2}$, indicating that the as-prepared material contains amorphous ceria. Increasing laser power causes local crystallization and the signature band of crystalline $\mathrm{CeO}_{2}$ appears (Fig. 6B) [35]. The Raman spectrum of the material after the thermal treatment (Fig. S11) reveals large presence of $\mathrm{CeO}_{2}$, while no obvious signal attributed to $\mathrm{Ce}^{3+}$ is noted (although the presence of $\mathrm{Ce}^{3+}$ cannot be ruled out on the basis of the Raman, as the signal may be comparatively much lower).

As in the case of the $\mathrm{TiO}_{2}$-based material, TEM shows that the resulting nanohybrid structure reveals a uniform coverage of the CNT backbone with the inorganic matter. However, while the Fe@CNTs/Pd@CeO 2 -magn sample does not present any free standing inorganic aggregate following a thorough TEM inspection (Fig. 7A-B), the Fe@CNTs/Pd@CeO ${ }_{2}$-filt catalyst isolation is accompanied by a relatively high density of islands of free standing Pd@CeO 2. (Fig. 7C-D). 

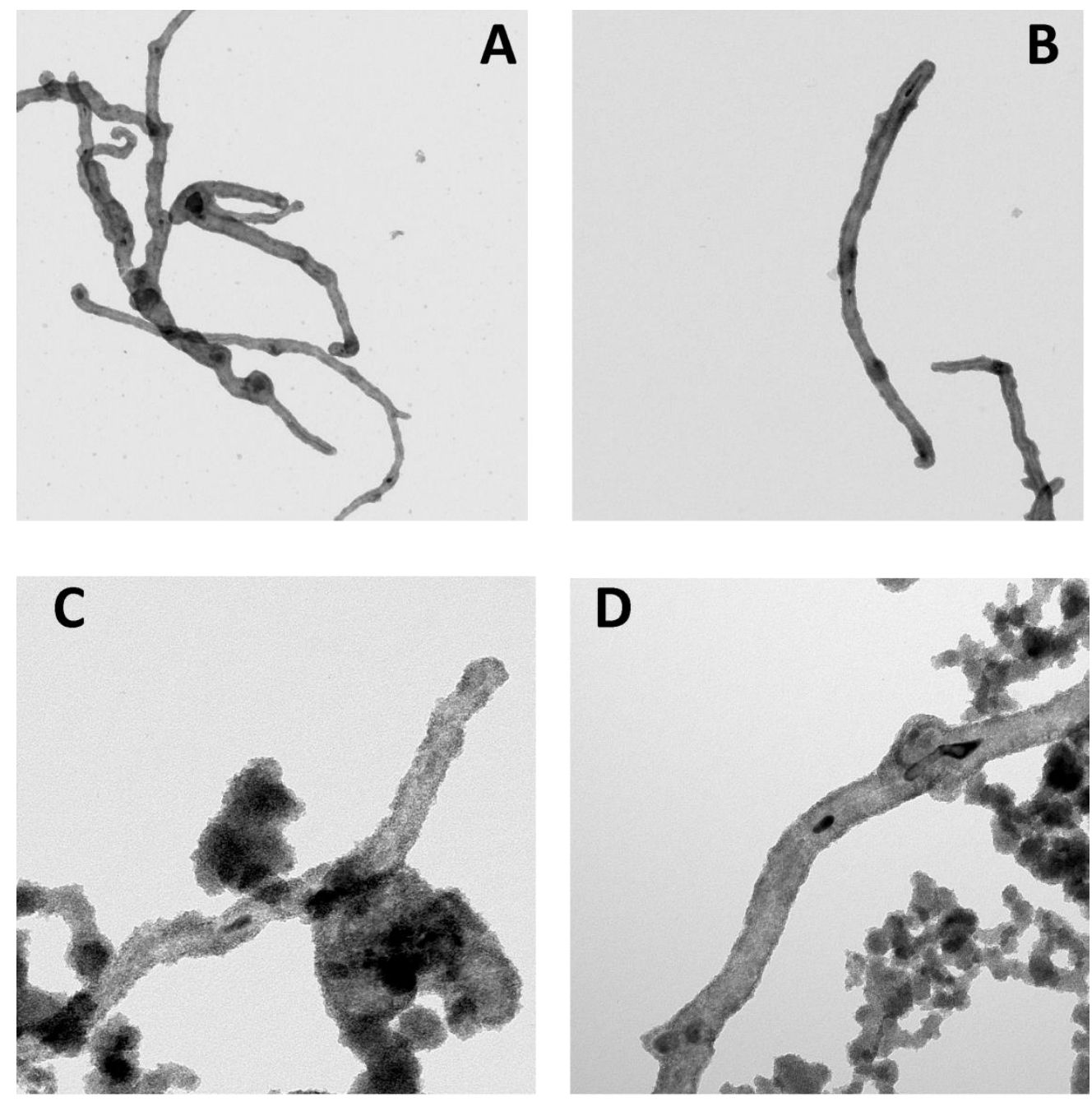

Fig. 7. A) and B) Representative TEM images of Fe@CNTs/Pd@CeO homogeneity of the material; C) and D) Representative TEM images of Fe@CNTs/Pd@CeO2-filt showing presence of free standing aggregates made of $\mathrm{Pd} @ \mathrm{CeO}_{2}$.

CO chemisorption has been employed to evaluate $\mathrm{Pd}$ accessibility after thermal treatment of the samples under $\mathrm{H}_{2} / \mathrm{Ar}$ atmosphere. The analysis revealed a CO/Pd ratio of 0.271 for $\mathrm{Fe} @ \mathrm{CNTs} / \mathrm{Pd} @ \mathrm{CeO}_{2}-$ magn and of 0.241 for Fe@CNTs/Pd@CeO 2 -filt. These values are in good agreement with what was observed for other prepared materials starting for the $\mathrm{Pd} @ \mathrm{CeO}_{2}$ core-shell units supported on either silanized $\mathrm{Al}_{2} \mathrm{O}_{3}$ [4] or decorating CNT [8]. Since comparable Pd accessibility has been observed for both the materials, the optimal organization of the Pd@CeO 2 units around the CNTs scaffold does not seem to be crucial to obtain a good accessibility of the Pd active phase. 
The activity of the present materials with respect to the WGSR process has been evaluated analysing the CO conversion rate at different temperatures $\left(200,250,275,300{ }^{\circ} \mathrm{C}\right)$, maintaining each thermal treatment for 4 hours. In the present case, the thermal pre-treatment of the two catalysts Fe@CNTs/Pd@CeO 2 -filt and Fe@CNTs/Pd@CeO 2 -magn was carried out in situ prior to the catalytic tests, by heating the material under $\mathrm{H}_{2} / \mathrm{Ar}$ flow at $250{ }^{\circ} \mathrm{C}$ for $1 \mathrm{~h}$. At each temperature, the catalysts displayed a stable CO conversion for the investigated time and, notably, an enhancement of the conversion rate of Fe@CNTs/Pd@CeO ${ }_{2}$-magn compared to Fe@CNTs/Pd@CeO ${ }_{2}$-filt was observed (Fig. 8). Remarkably, the gap in terms of CO conversion rate between both materials increases with the temperature in favor of the latter, the performances for the magnetically purified sample being almost doubled with respect to that filtered one at $300{ }^{\circ} \mathrm{C}$. No other by-products such as $\mathrm{CH}_{4}$ were revealed in $\mathrm{GC}$ analysis, indicating that hydrogenation processes are negligible under the present experimental conditions. In accordance with our previous results [11], the presence of the CNT in the catalyst formulation provides an improved

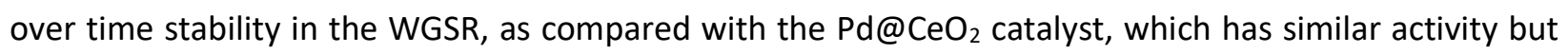
worse stability.

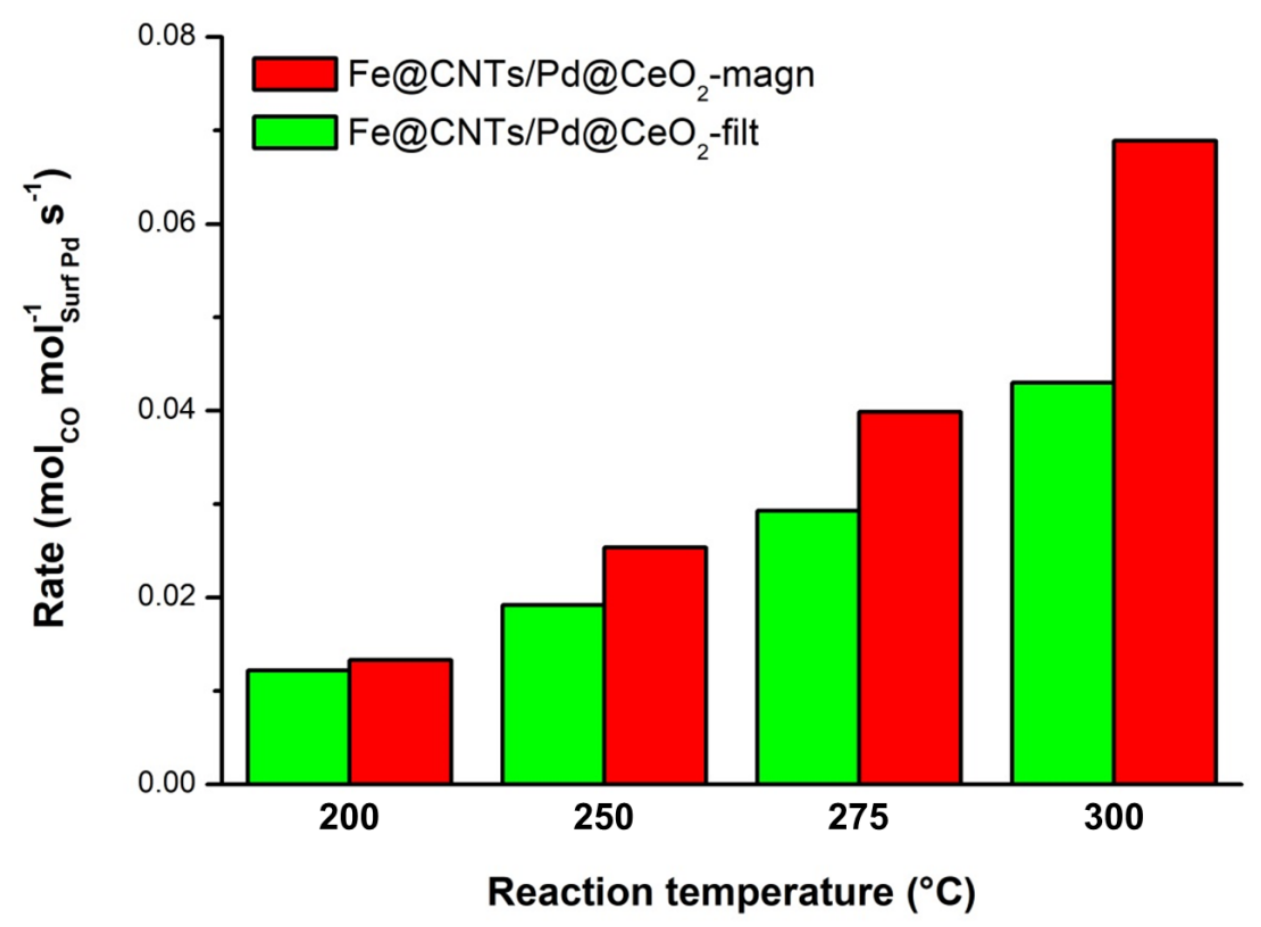

Fig. 8. Rate of $\mathrm{CO}$ conversion of $\mathrm{CeO}_{2}$-based materials at different temperatures normalized by the accessible Pd content. 
The present results highlight the importance of the optimization of the contact between the carbon nanomaterial and the inorganic active phase on the control of the activity of the final hybrid material.

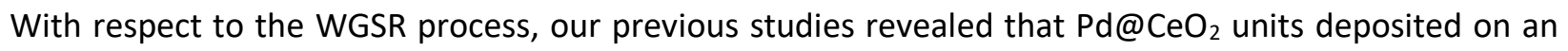
insulating support $\left(\mathrm{Al}_{2} \mathrm{O}_{3}\right)$ rapidly deactivates due to a combination of physical and electronic deactivation [36]: the $\mathrm{CO}$ adsorption on $\mathrm{Pd}$ nanoparticles is hindered after deep reduction of $\mathrm{CeO}_{2}$, by occlusion of the small mesopores after expansion of $\mathrm{CeO}_{2}$ crystal lattice and by electronic deactivation from reduced $\mathrm{CeO}_{2}$. The formation of hybrid composite materials employing oxidized CNT as scaffolds for directing the assembly of $\mathrm{Pd} @ \mathrm{CeO}_{2}$ units leads to an improved stability [34], although with a decreased activity. The improvement of stability was related with the electronic properties of the CNT scaffold that helps in draining the excess charge deriving from $\mathrm{CeO}_{2}$ reduction. A further development in terms of performances was obtained by ad hoc functionalization of CNTs to preserve their electronic conductivity and by applying a moderate thermal treatment to improve the textural properties of the composite [8]. The results presented in this study further confirm the importance of producing materials with an optimized intimate contact between the CNTs scaffold and the $\mathrm{Pd} @ \mathrm{CeO}_{2}$ matrix. In this case, the magnetic core provides a unique tool to purify CNTs homogeneously covered by $\mathrm{Pd@CeO}$ from the free standing inorganic phase, finally leading to the material with the more intimate contact between the two components.

\section{Conclusions}

The key role of CNTs in catalytic applications featuring nanostructured hybrid materials is directly demonstrated by utilizing a new concept based on magnetic sieving of the Fe-filled nanostructures. By this means, the final hybrid material is liberated of post-synthetic unreacted metallic matter that have so far represented a notorious problem, affecting intrinsic performances of the hybrid catalyst as well as the reproducibility of results. The new strategy, in contrast, allows for extremely homogeneous material where the contact $\mathrm{CNT} /$ metal oxide is maximized. In turn, this results in higher intrinsic performance, as demonstrated with two important catalytic processes such as photocatalytic hydrogen production (increase of $\sim 46 \%$ ) and WGSR (up to $\sim 45 \%$ for WGSR) for the two examples examined in this work. Our new approach has also the potential to mitigate the uncertainty of reported results involving CNT-based 
heterogeneous catalysts, as it provides a pathway to access structurally better defined hybrid, making future comparisons of activity more meaningful. Given the versatile preparative protocol and low-cost production of Fe-filled CNTs, we anticipate that the concept put forward in this paper can be adapted to prepare novel nanocatalysts for other heterogeneous processes.

\section{Acknowledgements}

The research leading to these results has received funding from the University of Trieste, INSTM, the Seventh Framework Programme [FP7/2007- 2013] under grant agreement $n^{\circ} 310651$ (SACS project). Dr. Emiliano Fonda and all the staff of SAMBA beamline (Synchrotron SOLEIL, France) are kindly acknowledged for assistance in measuring EXAFS spectra. DB thanks Cardiff University for generous funding. The Nanoscale and Microscale Research Centre (nmRC), University of Nottingham is acknowledged for access to electron microscopy instrumentation.

\section{Appendix A. Supplementary data}

\section{References}

[1] F. Wang, T.A. Shifa, X. Zhan, Y. Huang, K. Liu, Z. Cheng, C. Jiang, J. He, Recent advances in transitionmetal dichalcogenide based nanomaterials for water splitting, Nanoscale, 7 (2015) 19764-19788.

[2] P. Trogadas, V. Ramani, P. Strasser, T.F. Fuller, M.-O. Coppens, Hierarchically Structured Nanomaterials for Electrochemical Energy Conversion, Angew. Chem. Int. Ed., 55 (2016) 122-148.

[3] Y. Yamada, C.-K. Tsung, W. Huang, Z. Huo, S.E. Habas, T. Soejima, C.E. Aliaga, G.A. Somorjai, P. Yang, Nanocrystal bilayer for tandem catalysis, Nat. Chem., 3 (2011) 372-376.

[4] M. Cargnello, J.J.D. Jaén, J.C.H. Garrido, K. Bakhmutsky, T. Montini, J.J.C. Gámez, R.J. Gorte, P.

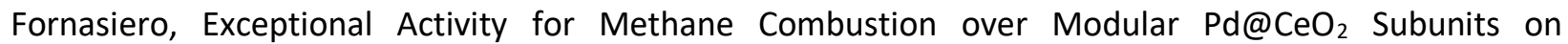
Functionalized $\mathrm{Al}_{2} \mathrm{O}_{3}$, Science, 337 (2012) 713-717.

[5] D.S. Su, S. Perathoner, G. Centi, Nanocarbons for the Development of Advanced Catalysts, Chem. Rev., 113 (2013) 5782-5816.

[6] D. Eder, Carbon Nanotube-Inorganic Hybrids, Chem. Rev., 110 (2010) 1348-1385.

[7] K. Woan, G. Pyrgiotakis, W. Sigmund, Photocatalytic Carbon-Nanotube- $\mathrm{TiO}_{2}$ Composites, Adv. Mater., 21 (2009) 2233-2239.

[8] A. Beltram, M. Melchionna, T. Montini, L. Nasi, R.J. Gorte, M. Prato, P. Fornasiero, Improved activity and stability of $\mathrm{Pd@CeO} 2$ core-shell catalysts hybridized with multi-walled carbon nanotubes in the water gas shift reaction, Catal. Today, 253 (2015) 142-148. 
[9] M. Melchionna, M. Bonchio, F. Paolucci, M. Prato, P. Fornasiero, Catalysis-material crosstalk at tailored nano-carbon interfaces, Top. Curr. Chem., 348 (2014) 139-180.

[10] A. Singh, A. Chandra, Significant Performance Enhancement in Asymmetric Supercapacitors based on Metal Oxides, Carbon nanotubes and Neutral Aqueous Electrolyte, Sci. Rep., 5 (2015) 15551.

[11] A. Beltram, M. Melchionna, T. Montini, L. Nasi, P. Fornasiero, M. Prato, Making $\mathrm{H}_{2}$ from light and biomass-derived alcohols: the outstanding activity of newly designed hierarchical $\mathrm{MWCNT}_{\mathrm{Pd}} @ \mathrm{TiO}_{2}$ hybrid catalysts, Green Chem., 19 (2017) 2379-2389.

[12] M.B. Gawande, R. Luque, R. Zboril, The Rise of Magnetically Recyclable Nanocatalysts, ChemCatChem, 6 (2014) 3312-3313.

[13] A. Schätz, T.R. Long, R.N. Grass, W.J. Stark, P.R. Hanson, O. Reiser, Immobilization on a Nanomagnetic Co/C Surface Using ROM Polymerization: Generation of a Hybrid Material as Support for a Recyclable Palladium Catalyst, Adv. Funct. Mater., 20 (2010) 4323-4328.

[14] S. Shylesh, V. Schünemann, W.R. Thiel, Magnetisch abtrennbare Nanokatalysatoren: Brücken zwischen homogener und heterogener Katalyse, Angew. Chem., 122 (2010) 3504-3537.

[15] V. Polshettiwar, R. Luque, A. Fihri, H. Zhu, M. Bouhrara, J.-M. Basset, Magnetically Recoverable Nanocatalysts, Chem. Rev., 111 (2011) 3036-3075.

[16] A. Stopin, F. Pineux, R. Marega, D. Bonifazi, Magnetically Active Carbon Nanotubes at Work, Chem. Eur. J., 21 (2015) 9288-9301.

[17] F. Pineux, R. Marega, A. Stopin, A. La Torre, Y. Garcia, E. Devlin, C. Michiels, A. N. Khlobystov, D. Bonifazi, Biotechnological promises of Fe-filled CNTs for cell shepherding and magnetic fluid hyperthermia applications, Nanoscale, 7 (2015) 20474-20488.

[18] R. Marega, F. De Leo, F. Pineux, J. Sgrignani, A. Magistrato, A.D. Naik, Y. Garcia, L. Flamant, C. Michiels, D. Bonifazi, Functionalized Fe-Filled Multiwalled Carbon Nanotubes as Multifunctional Scaffolds for Magnetization of Cancer Cells, Adv. Funct. Mater., 23 (2013) 3173-3184.

[19] M.V. Bracamonte, M. Melchionna, A. Stopin, A. Giulani, C. Tavagnacco, Y. Garcia, P. Fornasiero, D. Bonifazi, M. Prato, Carboxylated, Fe-filled multiwalled carbon nanotubes as versatile catalysts for $\mathrm{O}_{2}$ reduction and $\mathrm{H}_{2}$ evolution reactions at physiological pH, Chem.Eur. J., 21 (2015) 12769-12777.

[20] D. Deng, L. Yu, X. Chen, G. Wang, L. Jin, X. Pan, J. Deng, G. Sun, X. Bao, Iron Encapsulated within Pod-like Carbon Nanotubes for Oxygen Reduction Reaction, Angew. Chem. Int. Ed., 52 (2013) 371-375.

[21] X. Yang, H. Yu, F. Peng, H. Wang, Confined Iron Nanowires Enhance the Catalytic Activity of Carbon Nanotubes in the Aerobic Oxidation of Cyclohexane, ChemSusChem, 5 (2012) 1213-1217.

[22] K. Bakhmutsky, N.L. Wieder, M. Cargnello, B. Galloway, P. Fornasiero, R.J. Gorte, A versatile route to core-shell catalysts: synthesis of dispersible $\mathrm{M} @$ oxide $\left(\mathrm{M}=\mathrm{Pd}, \mathrm{Pt}\right.$; oxide $\left.=\mathrm{TiO}_{2}, \mathrm{ZrO}_{2}\right)$ nanostructures by self-assembly, ChemSusChem, 5 (2012) 140-148. 
[23] M. Cargnello, N.L. Wieder, T. Montini, R.J. Gorte, P. Fornasiero, Synthesis of dispersible ${\mathrm{Pd} @ \mathrm{CeO}_{2}}$ core-shell nanostructures by self-assembly, J. Am. Chem. Soc., 132 (2010) 1402-1409.

[24] M. Tella, M. Auffan, L. Brousset, J. Issartel, I. Kieffer, C. Pailles, E. Morel, C. Santaella, B. Angeletti, E. Artells, J. Rose, A. Thiéry, J.-Y. Bottero, Transfer, Transformation, and Impacts of Ceria Nanomaterials in Aquatic Mesocosms Simulating a Pond Ecosystem, Environ. Sci. Technol., 48 (2014) 9004-9013.

[25] V. Briois, E. Fonda, S. Belin, L. Barthe, C. La Fontaine, F. Langlois, M. Ribbens, F. Villain, SAMBA: The 4-40 keV X-ray absorption spectroscopy beamline at SOLEIL, UVX 2010 - 10e Colloque sur les Sources Cohérentes et Incohérentes UV, VUV et X ; Applications et Développements Récents, (2011) 41-47.

[26] X. Liu, G. Zhu, X. Wang, X. Yuan, T. Lin, F. Huang, Progress in Black Titania: A New Material for Advanced Photocatalysis, Adv. Energy Mater., 6 (2016) 1600452.

[27] X. Chen, L. Liu, F. Huang, Black titanium dioxide $\left(\mathrm{TiO}_{2}\right)$ nanomaterials, Chem. Soc. Rev., 44 (2015) 1861-1885.

[28] A. Naldoni, M. Allieta, S. Santangelo, M. Marelli, F. Fabbri, S. Cappelli, C.L. Bianchi, R. Psaro, V. Dal Santo, Effect of Nature and Location of Defects on Bandgap Narrowing in Black $\mathrm{TiO}_{2}$ Nanoparticles, J. Am. Chem. Soc., 134 (2012) 7600-7603.

[29] O. Frank, M. Zukalova, B. Laskova, J. Kurti, J. Koltai, L. Kavan, Raman spectra of titanium dioxide (anatase, rutile) with identified oxygen isotopes $(16,17,18)$, Phys. Chem. Chem. Phys., 14 (2012) 1456714572.

[30] T. Ohsaka, F. Izumi, Y. Fujiki, Raman spectrum of anatase, $\mathrm{TiO}_{2}$, J. Raman Spectrosc., 7 (1978) 321324.

[31] K.S.W. Sing, Reporting physisorption data for gas/solid systems with special reference to the determination of surface area and porosity (Recommendations 1984), Pure Appl. Chem. , 1985, pp. 603.

[32] C.P. Lin, H. Chen, A. Nakaruk, P. Koshy, C.C. Sorrell, Effect of Annealing Temperature on the Photocatalytic Activity of $\mathrm{TiO}_{2}$ Thin Films, Energy Procedia, 34 (2013) 627-636.

[33] T. Montini, M. Melchionna, M. Monai, P. Fornasiero, Fundamentals and Catalytic Applications of $\mathrm{CeO}_{2}$-Based Materials, Chem. Rev., 116 (2016) 5987-6041.

[34] M. Cargnello, M. Grzelczak, B. Rodríguez-González, Z. Syrgiannis, K. Bakhmutsky, V. La Parola, L.M. Liz-Marzán, R.J. Gorte, M. Prato, P. Fornasiero, Multiwalled Carbon Nanotubes Drive the Activity of Metal@oxide Core-Shell Catalysts in Modular Nanocomposites, J. Am. Chem. Soc., 134 (2012) 1176011766.

[35] J.E. Spanier, R.D. Robinson, F. Zhang, S.-W. Chan, I.P. Herman, Size-dependent properties of $\mathrm{CeO}_{2-y}$ nanoparticles as studied by Raman scattering, Phys. Rev. B, 64 (2001) 245407.

[36] N.L. Wieder, M. Cargnello, K. Bakhmutsky, T. Montini, P. Fornasiero, R.J. Gorte, Study of the WaterGas-Shift Reaction on Pd@ $@ \mathrm{CeO}_{2} / \mathrm{Al}_{2} \mathrm{O}_{3}$ Core-Shell Catalysts, J. Phys. Chem. C, 115 (2011) 915-919. 
